\title{
On Path Correlation And PERT Bias
}

Arunava Banerjee ${ }^{1}$

Anand Paul ${ }^{2}$

March 2007

${ }^{1}$ Arunava Banerjee, Department of Computer and Information Science and Engineering, University of Florida, CSE Bldg, Room 301, P.O.Box 116120, Gainesville, Fl 32611

Email address: arunava@cise.ufl.edu

${ }^{2}$ Anand Paul, Department of Decision and Information Sciences, Warrington College of Business Administration, University of Florida, 333 Stuzin Hall, P.O.Box 117169, Gainesville, Fl 32611 Email address: paulaa@ufl.edu (Corresponding Author) 


\title{
On Path Correlation And PERT Bias
}

\begin{abstract}
$\underline{\text { Abstract }}$
Most studies of project time estimation assume that (a) activity times are mutually independent random variables; many also assume that (b) path completion times are mutually independent. In this paper, we subject the impact of both these assumptions to close scrutiny. Using tools from multivariate analysis, we make a theoretical study of the direction of the error in the classical PERT method of estimating mean project completion time when correlation is ignored. We also investigate the effect of activity dependence on the normality of path length via simulation.
\end{abstract}

Keywords: Project management, PERT, correlation, project networks, Slepian's inequality. 


\section{On Path Correlation and PERT Bias}

\section{Introduction}

The critical path method of estimating project completion time and its probabilistic version, PERT, are widely used by project management practitioners. It is important for practitioners to have sound a priori estimates of project completion time because cost planning and resource allocation decisions hinge crucially on these estimates.

Ever since the 1950's, when the PERT method was formulated, researchers have attempted to construct a rigorous theoretical foundation for it. It is now well accepted that PERT gives useful estimates. However, there is still work to be done on isolating potential sources of bias in its application and on developing guidelines linking specific assumptions to their impact on PERT bias. The present paper studies correlation between activities and between paths as sources of PERT bias. For comprehensive surveys of research on project time estimation, see Elmaghraby [3] and Slowinski \& Weglarz [11], among many other sources.

Most studies of project time estimation assume that (a) activity times are mutually independent random variables; many also assume that (b) path completion times are mutually independent. In this note, we study PERT bias in projects with correlated activities and paths using tools from multivariate analysis. We also report on a simulation experiment that sheds some light on the effect of activity dependence on the normality of path length distribution. 


\section{The impact of correlation on PERT bias}

Recall that the PERT method estimates the mean project completion to be $\operatorname{Max}\left(\mathrm{EL}_{1}, \ldots, \mathrm{EL}_{\mathrm{N}}\right)$ where $\mathrm{L}_{\mathrm{i}}$ is the length of path ' $\mathrm{i}$ ' in the project network; this is always smaller than $\mathrm{E}$ Max $\left(\mathrm{L}_{1}, \ldots, \mathrm{L}_{\mathrm{N}}\right)$, the true mean project completion time. The difference between these two metrics is the magnitude of PERT bias. The PERT method therefore always underestimates true mean project duration, a fact that is widely known. Colloquially speaking, PERT yields optimistic estimates. MacCrimmon and Ryavec [7] (hereafter referred to as M\&R) inferred from numerical examples that the higher the correlation in a network because of overlapping paths, the smaller the magnitude of PERT bias, but this conclusion has not, to our knowledge, been subjected to rigorous scrutiny. We show that whether or not this rule of thumb is true depends on the nature of the correlation between activities and paths, and on the distribution of path lengths and activity durations.

M\&R used the following example to illustrate their point:

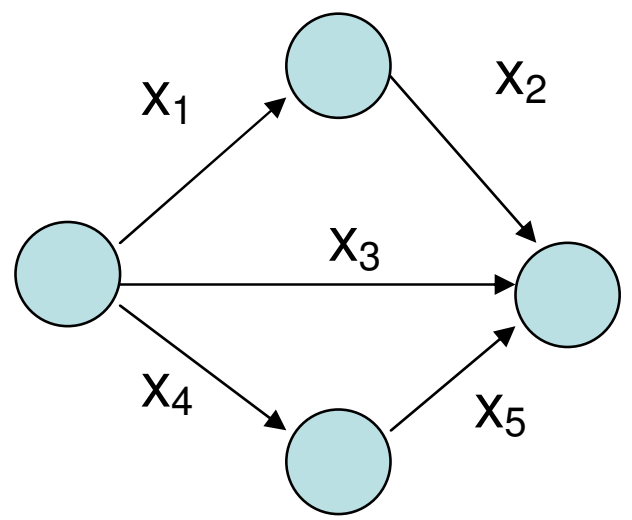

Project A

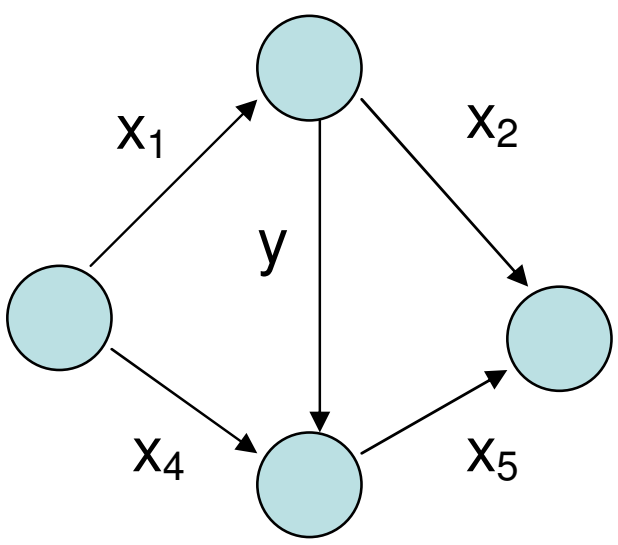

Project B

\section{Figure 1}


In Figure 1, the path lengths in Project A are the following:

Path 1: $\mathrm{x}_{1}+\mathrm{x}_{2}$

Path 2: $x_{3}$

Path 3: $\mathrm{x}_{4}+\mathrm{x}_{5}$

Note that the path lengths are independent random variables if all the activities are mutually independent. The path lengths in Project B are as follows:

Path 1: $\mathrm{x}_{1}+\mathrm{x}_{2}$

Path 2: $\mathrm{x}_{1}+\mathrm{y}+\mathrm{x}_{5}$

Path 3: $\mathrm{x}_{4}+\mathrm{x}_{5}$

Note that although the projects have two path lengths that are identical, the two projects differ in an essential way: the path lengths in Project B are pairwise dependent because of the connecting activity with duration $y . M \& R$ conjecture that the correlated paths in Project B should buy it a smaller value of PERT bias compared with Project A provided the mean lengths of Path 2 are the same in both projects. They verify their conjecture with specific discrete distributions for the activity times. However, the following counterexample shows that even for the specific networks constructed by M\&R, a correlated project may carry a higher PERT bias than an independent project.

We fix the activity times to the following values: $x_{1}=2$ with probability $1 / 2$ and 4 with probability $1 / 2, x_{2}=6, x_{3}=5, x_{4}=4, x_{5}=5, y=0$ with probability $1 / 2$ and 2 with probability $1 / 2$. Note that Path 1 and Path 3 in Project A are identical to the corresponding paths in Project B; Path 2 in Project A has the same mean completion time as Path 2 in Project B. A simple computation shows that the completion time of Project $A$ is 9 with probability $1 / 2$ and 10 with probability $1 / 2$, giving a mean completion time of 9.5. On the other hand, the completion time of Project B is 9 with 
probability $1 / 2,10$ with probability $1 / 4$ and 11 with probability $1 / 4$ giving a mean completion time of 9.75. This example shows that correlation between paths may inflate PERT bias.

In the remainder of the paper, we attempt to find broad patterns between PERT bias and correlation. We deal separately with two categories of projects: (a) projects with independent activities giving rise to correlated paths, and (b) projects in which the activities are correlated per se.

\subsection{Projects with independent activities}

We begin with a result on PERT bias in networks with independent activities and overlapping paths (that is, paths with at least one activity in common). Although we have not seen it explicitly stated in the literature, it may be easily inferred from the theoretical framework developed in Dodin [2]. The proof is a direct application of some properties of associated random vectors. A random vector $\mathbf{X}=\left(X_{1}, \ldots, X_{N}\right)$ is said to be associated if $\operatorname{Cov}(f(X), g(X)) \geq 0$ for all coordinatewise increasing functions $\mathrm{f}($.$) and \mathrm{g}\left(\right.$.) from $R^{N}$ to $\mathrm{R}$. If $\mathbf{X}$ is associated, it follows from the definition that the components of $\mathbf{X}$ are pair-wise positively correlated. The other facts about associated random vectors used in the proof of the following theorem are taken from Esary, Proschan and Walkup [4].

\section{Theorem 1}

Consider a project network with overlapping paths in which the activity durations are mutually independent random variables with arbitrary distributions. Then the path lengths are positively correlated, and the true mean project completion time is smaller than the mean project completion time computed under the assumption that the path lengths are independent rather than positively correlated. 
Proof: First we claim that if the activity durations are mutually independent, the path lengths Ti constitute an associated random vector. This is a consequence of the following two facts: (1) A random vector consisting of independent components is associated; (2) If $\mathbf{T}$ is associated and $f_{1}, \ldots, f_{K}$ are coordinate-wise non-decreasing real valued functions of $\mathbf{T}$, then the random vector $\left(\mathrm{f}_{1}(\mathbf{T}), \ldots, \mathrm{f}_{\mathrm{K}}(\mathbf{T})\right)$ is associated. Consider a project network with $\mathrm{K}$ independent paths and $\mathrm{N}$ independent activities with durations $\mathrm{T}_{1}, \ldots, \mathrm{T}_{\mathrm{N}}$. By $(1)$ above, $\left(\mathrm{T}_{1}, \ldots, \mathrm{T}_{\mathrm{N}}\right)$ is associated. It follows from (2) that the collection of path lengths is an associated random vector as claimed. Now the theorem follows from the following property of associated random vectors:

$$
\operatorname{Pr}\left\{T_{1} \leq t_{1}, \ldots, T_{K} \leq t_{K}\right\} \geq \prod_{i=1}^{K} \operatorname{Pr}\left\{T_{i} \leq t_{i}\right\} . \quad \text { Q.E.D. }
$$

Remark 1: The proof is not valid in general when the activity durations are correlated. However, if the activity durations are normally distributed, the result does hold even when activities are correlated. This is because normal random variables are positively correlated if and only if they are associated (Pitt [7]).

Theorem 1 compares two extreme categories: independent paths and positively correlated paths. A natural question to ask is whether the result can be extended to account for all possible degrees of correlation between paths. Our analysis of the M\&R project networks shows that one cannot expect to obtain a structural result in this direction if the project networks under comparison have different topologies. Let us therefore fix the topology of a project network and see if we can quantify the impact of an increase in correlation between paths. It is immediate that the covariance between two paths is equal to the sum of the variances of the activities common to both paths. So we can model an increase in correlation between two paths by inflating the variance of a common 
activity while keeping its mean unchanged. It follows easily from standard results on convex stochastic ordering that this will actually increase PERT bias for many distributional classes of activity times (eg. Normal, Uniform, and most location-scale distributions).

\subsection{Projects with correlated activities}

Real world projects consist of a number of activities, the durations of some of which are correlated with the durations of others. For example, if the same subcontractor is responsible for completing a number of related activities that share the same resources (for instance, the same construction crew or the same machines), it is reasonable to conclude that the activity durations of the subset of tasks covered by the subcontractor are positively correlated (if the contractor is uniformly efficient or inefficient across all the activities under his purview) or negatively correlated (if he has limited resources and the efficient accomplishment of some activities hinders the efficient completion of others). For project networks incorporating complex interdependencies between activities stemming from resource sharing, the covariance between two paths is not simply equal to the sum of the variances of the activities common to both paths. It is interesting to see whether we can deepen Theorem 1 by relaxing the activity independence assumption by allowing activity durations to be correlated. We provide a partial answer in Theorem 2 below. The price paid for relaxing activity independence is that the result we obtain is no longer distribution free.

\section{Theorem 2}

For a PERT network with a multivariate normal distribution of activity durations and fixed mean and variance vectors, the greater the correlation between activity durations, the smaller is the error in the PERT method. 
Proof: Let $\mathbf{A}=\left(\mathrm{A}_{1}, \mathrm{~A}_{2}, \ldots, \mathrm{A}_{\mathrm{N}}\right)$ and $\mathbf{B}=\left(\mathrm{B}_{1}, \mathrm{~B}_{2}, \ldots, \mathrm{B}_{\mathrm{N}}\right)$ be multivariate normal vectors of activity times with the same mean vector $(\mu)$ and covariance matrices $\sum_{A}=\left(\sigma_{i j}^{A}\right)$ and $\sum_{B}=\left(\sigma_{i j}^{B}\right)$.

Consider an $\mathrm{N}$-activity network with a given topology; we obtain two versions of this network by endowing it with activity vectors $\mathbf{A}$ and $\mathbf{B}$, respectively. Let $\mathbf{L}=\left(\mathrm{L}_{1}, \mathrm{~L}_{2}, \ldots, \mathrm{L}_{\mathrm{K}}\right)$ be the vector of path lengths corresponding to activity vector $\mathbf{A}$ and $\mathbf{M}=\left(\mathrm{M}_{1}, \mathrm{M}_{2}, \ldots, \mathrm{M}_{\mathrm{K}}\right)$ be the vector of path lengths corresponding to activity vector $\mathbf{B}$. Since each path length is a sum of activity durations and since the two networks have the same topology, it follows that there exists a linear operator $\mathbf{Q}$ (characterized by a $N$ by $K$ matrix) such that $\mathbf{L}=\mathbf{A Q}$ and $\mathbf{M}=\mathbf{B Q}$. Now since $\mathbf{A}$ and $\mathbf{B}$ are multivariate normal vectors with the same mean and variance vectors and $\mathbf{Q}$ is a linear operator, it follows from a standard property of the multivariate normal distribution that $\mathbf{L}$ and $\mathbf{M}$ are multivariate normal vectors too, and have common mean and variance vectors. Further, if each off-diagonal (covariance) term in the covariance matrix of $\mathbf{B}$ is greater than or equal to the corresponding off-diagonal term in the covariance matrix of $\mathbf{A}$ (and the variance terms are identical), it follows that each off-diagonal term in the covariance matrix of $\mathbf{M}$ is greater than or equal to the corresponding off-diagonal term in the covariance matrix of $\mathbf{L}$ (and again, the variance terms are identical). Finally, we recall Slepian's inequality (see Joag-Dev, Perlman and Pitt [4]) which states that in the case of a multivariate normal vector $\left(\mathrm{X}_{1}, \ldots, \mathrm{X}_{\mathrm{N}}\right)$ with $n$ by $n$ covariance matrix $\sum=\left(\sigma_{i j}\right), \operatorname{Pr}\left\{\operatorname{Max}\left(\mathrm{X}_{1}, \ldots, \mathrm{X}_{\mathrm{N}}\right) \leq \mathrm{C}\right\}$ is increasing in each $\sigma_{i j}($ with $\mathrm{i}<\mathrm{j})$ for all C. The theorem now follows from the following steps:

$\operatorname{EMax}\left(\mathrm{M}_{1}, \ldots, \mathrm{M}_{\mathrm{N}}\right)=\int_{0}^{\infty} \operatorname{Pr}\left\{\operatorname{Max}\left(M_{1}, \ldots, M_{N}\right)>x\right\} d x=\int_{0}^{\infty} 1-\operatorname{Pr}\left\{\operatorname{Max}\left(M_{1}, \ldots, M_{N}\right) \leq x\right\} d x$ $\leq \int_{0}^{\infty} 1-\operatorname{Pr}\left\{\operatorname{Max}\left(L_{1}, \ldots, L_{N}\right) \leq x\right\} d x=\mathrm{E} \operatorname{Max}\left(\mathrm{L}_{1}, \ldots, \mathrm{L}_{\mathrm{N}}\right) \quad$ Q.E.D. 


\section{Corollary 3}

Consider a project network in which the vector of activity durations is multivariate normal and the activity durations are pairwise positively correlated.

(a) Then the true mean project completion time is smaller than the mean project completion time computed under the assumption that the activity durations are independent rather than positively correlated.

(b) The probability that the project is completed within a given due date is greater with positively correlated activities than with independent activities.

Remark 2: Rather than assume a multivariate normal vector of activity durations, we might simplify the set up of Theorem 2 by starting with a multivariate normal vector of path lengths. This is the framework that is usually adopted for the PERT method with the difference that PERT imposes a particular correlation structure on the multivariate normal distribution of path lengths: the special assumption of independence (which is equivalent to zero correlation for the normal distribution). Restated along these lines, the proof of Theorem 2 would be quicker in that we would not need to start with vectors of activity durations and transform them into vectors of path lengths via linear operators. However, the proof in the existing form is instructive because it brings out the special properties of the multivariate normal distribution that are necessary for the proof to work. Indeed, the linear transformation used in the proof would change the distributions of many multivariate random vectors.

Remark 3: Theorem 2 implies that when path lengths form a pair-wise positively correlated normal vector, the optimistic bias in the PERT method is in truth smaller than the bias when path 
lengths form an independent normal vector. This is the analogue of Theorem 1 for networks with correlated activities.

\subsection{Extension to non-normal distributions}

It is pertinent to ask whether Theorem 2 can be extended to distributions other than the multivariate normal. We give a simple extension in which path lengths are positively correlated but the marginal distributions are not normally distributed and have finite support. Consider an Ndimensional sphere with probability mass uniformly distributed in it. We label the coordinates of a random point in the sphere $\mathrm{U}_{1}, \ldots, \mathrm{U}_{\mathrm{N}}$. Consider the $\mathrm{M}$ random variables defined as follows:

$\mathrm{X}_{1}=\mathrm{a}_{11} \mathrm{U}_{1}+\ldots+\mathrm{a}_{1 \mathrm{~N}} \mathrm{U}_{\mathrm{N}}$

$\mathrm{X}_{2}=\mathrm{a}_{21} \mathrm{U}_{1}+\ldots+\mathrm{a}_{2 \mathrm{~N}} \mathrm{U}_{\mathrm{N}}$

$\mathrm{X}_{\mathrm{M}}=\mathrm{a}_{\mathrm{M} 1} \mathrm{U}_{1}+\ldots+\mathrm{a}_{\mathrm{MN}} \mathrm{U}_{\mathrm{N}}$

where $\mathrm{a}_{\mathrm{ij}}$ are positive numbers.

We can use these random variables to model $\mathrm{M}$ positively correlated paths in a project network. 


\section{Scatter Plot of Samples and corresponding Marginal Distributions}

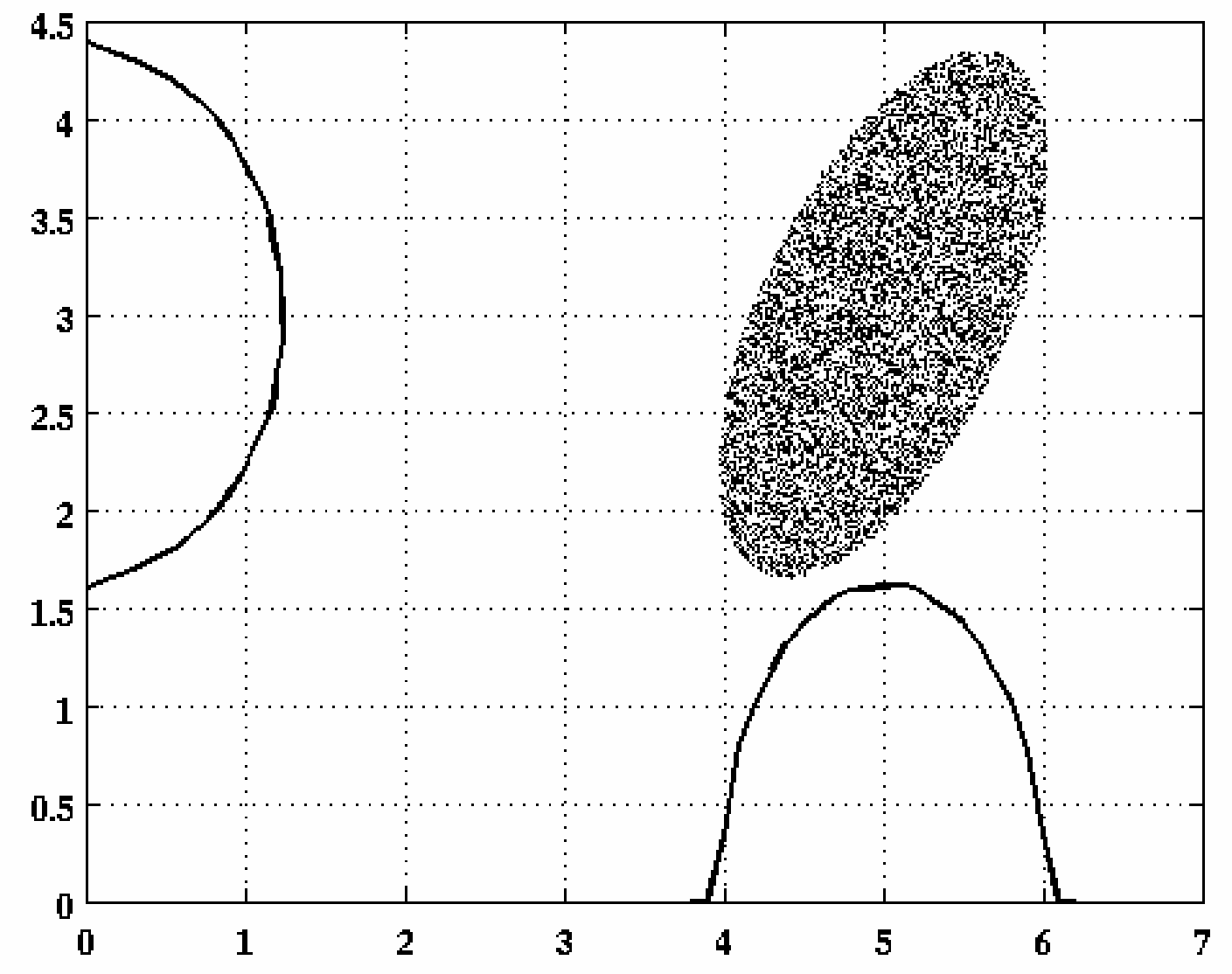

\section{Figure 2}

Now Slepian's inequality applies to the random vector $\left(\mathrm{X}_{1}, \ldots, \mathrm{X}_{\mathrm{M}}\right)$ since the distribution of $\left(\mathrm{X}_{1}, \ldots, \mathrm{X}_{\mathrm{M}}\right)$ can be easily shown to be a member of the class of elliptically contoured distributions, to which Slepian's inequality has been proved to apply (see [6]). The marginal distributions of $X_{\mathrm{i}}$ are symmetric and unimodal; this follows from the fact that the underlying generating distribution is radially symmetric since it is uniformly distributed in a sphere. Figure 2 shows joint distribution of activity times and the marginal distributions from simulations for the bivariate case. Note that both marginal distributions have finite support. 
We note in passing that Slepian's Inequality (and therefore Theorem 2) does not apply when we replace the normally distributed marginal distributions by normal distributions truncated at zero. An essential requirement for the inequality to hold is that the joint distribution be isotropic; truncating the marginals at zero destroys the spatial symmetry of the joint distribution because the truncation is one-sided and hence asymmetric.

\subsection{The case of highly correlated paths}

There is one situation in which the rule "greater correlation implies smaller bias" does hold. This is the case when the correlation between the paths in a project network is sufficiently high. To show this formally, consider two projects A and B each with 2 parallel paths (the result can easily be generalized to an arbitrary number of paths, as we shall show). Denote the paths of project A by $\mathrm{X}_{1}$ and $\mathrm{X}_{2}$ and the paths of project $\mathrm{B}$ by $\mathrm{Y}_{1}$ and $\mathrm{Y}_{2}$.

We write the distributions of the path lengths of projects A and B as follows:

$\mathrm{X}_{1}=\mathrm{a}_{11} \mathrm{Z}_{1}+\ldots+\mathrm{a}_{1 \mathrm{~N}} \mathrm{Z}_{\mathrm{N}}$

$\mathrm{X}_{2}=\mathrm{a}_{21} \mathrm{Z}_{1}+\ldots+\mathrm{a}_{2 \mathrm{~N}} \mathrm{Z}_{\mathrm{N}}$

$\mathrm{Y}_{1}=\mathrm{b}_{11} \mathrm{Z}_{1}+\ldots+\mathrm{b}_{1 \mathrm{~N}} \mathrm{Z}_{\mathrm{N}}$

$\mathrm{Y}_{2}=\mathrm{b}_{21} \mathrm{Z}_{1}+\ldots+\mathrm{b}_{2 \mathrm{~N}} \mathrm{Z}_{\mathrm{N}}$

where the $\mathrm{Z}_{\mathrm{i}}$ are independently and identically distributed random variables. With this set up, we can model large classes of distributions $\mathrm{X}_{1}, \mathrm{X}_{2}, \mathrm{Y}_{1}$ and $\mathrm{Y}_{2}$ and simultaneously control the correlation between the pairs $\left(\mathrm{X}_{1}, \mathrm{X}_{2}\right)$ and $\left(\mathrm{Y}_{1}, \mathrm{Y}_{2}\right)$. We may assume $\mathrm{E}\left(\mathrm{Z}_{\mathrm{i}}\right)=0$ without any loss of generality; we can always add a non-zero bias term to each path length to shift it if needed. We denote the variance of $\mathrm{Z}_{\mathrm{i}}$ by $\mathrm{V}$.

Consider the matrices $\mathrm{A}=\left(\mathrm{a}_{\mathrm{ij}}\right)$ and $\mathrm{B}=\left(\mathrm{b}_{\mathrm{ij}}\right)$. It can easily be verified that 
Variance $\mathrm{X}_{1}=\mathrm{V} \sum_{j=1}^{N} a_{1 j}^{2}$, Variance $\mathrm{X}_{2}=\mathrm{V} \sum_{j=1}^{N} a_{2 j}^{2}$, Variance $\mathrm{Y}_{1}=\mathrm{V} \sum_{j=1}^{N} b_{1 j}^{2}$, Variance $\mathrm{Y}_{2}=\mathrm{V} \sum_{j=1}^{N} b_{2 j}^{2}$ Covariance $\left(\mathrm{X}_{1}, \mathrm{X}_{2}\right)=\mathrm{V} \sum_{j=1}^{N} a_{1 j} a_{2 j}$, and Covariance $\left(\mathrm{Y}_{1}, \mathrm{Y}_{2}\right)=\mathrm{V} \sum_{j=1}^{N} b_{1 j} b_{2 j}$.

We may regard the rows of matrices A and B as vectors in N-dimensional Euclidean space. Note that the variance of each $\mathrm{X}_{\mathrm{i}}$ and $\mathrm{Y}_{\mathrm{j}}$ is a fixed multiple of the square of the length of the $\mathrm{N}$-vector representing it. So the condition that the two projects have paths with the same variance implies that $\mathrm{A}$ and $\mathrm{B}$ have rows that represent vectors of the same Euclidean length, row for row. From the formulas developed above, it is clear that the covariance between the paths of project $\mathrm{A}$ is greater than the covariance between the paths of project B if and only if the dot product between the rows of the matrix $\mathrm{A}$ is greater than the dot product between the rows of the matrix $\mathrm{B}$. Now recall that that the dot product of two N-vectors $\mathrm{X}_{\mathrm{i}}$ and $\mathrm{X}_{\mathrm{j}}$ is $\left|X_{i} \| X_{j}\right| \operatorname{Cos}\left(\vartheta_{i j}{ }^{X}\right)$ where $\vartheta_{i j}{ }^{X}$ is the angle between the two vectors. Since $\left|X_{i}\right|=\left|Y_{i}\right|$, the condition Covariance $\left(\mathrm{X}_{1}, \mathrm{X}_{2}\right)>$ Covariance $\left(\mathrm{Y}_{1}, \mathrm{Y}_{2}\right)$ is equivalent to $\operatorname{Cos}\left(\vartheta_{12}{ }^{X}\right)>\operatorname{Cos}\left(\vartheta_{12}{ }^{Y}\right) \Leftrightarrow \vartheta_{12}{ }^{X}<\vartheta_{12}{ }^{Y}$.

Now Variance $\mathrm{X}_{\mathrm{i}}=$ Variance $\mathrm{Y}_{\mathrm{i}}(\mathrm{i}=1,2)$ and Covariance $\left(\mathrm{X}_{1}, \mathrm{X}_{2}\right)>$ Covariance $\left(\mathrm{Y}_{1}, \mathrm{Y}_{2}\right)$ does not necessarily imply that $\mathrm{E} \operatorname{Max}\left(\mathrm{X}_{1}, \mathrm{X}_{2}\right)<\mathrm{E} \operatorname{Max}\left(\mathrm{Y}_{1}, \mathrm{Y}_{2}\right)$. This can be verified with the following matrices:

$$
\left[\begin{array}{ll}
0 & 5 \\
\sqrt{24.99} & 5.1
\end{array}\right]=A \quad\left[\begin{array}{cc}
4 & 3 \\
1 & 7
\end{array}\right]=B
$$

and $\mathrm{Z}_{\mathrm{i}}$ uniformly distributed on $[-0.5,0.5]$. Note that the correlation coefficients of $\left(\mathrm{X}_{1}, \mathrm{X}_{2}\right)$ and $\left(\mathrm{Y}_{1}, \mathrm{Y}_{2}\right)$ are 0.72 and 0.707 , respectively. In this case it can be verified that $\operatorname{EMax}\left(\mathrm{X}_{1}, \mathrm{X}_{2}\right)=0.612>\mathrm{E} \operatorname{Max}\left(\mathrm{Y}_{1}, \mathrm{Y}_{2}\right)=0.594$ 
However, we can salvage the following result, stated for the bivariate case but easily extended to joint distributions of $\mathrm{N}$ random variables.

\section{Theorem 4}

Fix the correlation coefficient of $Y_{1}$ and $Y_{2}$ to any value $\rho_{0}$ strictly less than 1 and let

Variance $X_{i}=$ Variance $Y_{i}(i=1,2)$. Then there exists a positive fraction $C\left(\rho_{0}\right)$ such that if the correlation coefficient of $X_{1}$ and $X_{2}$ is greater than $C\left(\rho_{0}\right)$, it follows that $E \operatorname{Max}\left(X_{1}, X_{2}\right)<$ $E \operatorname{Max}\left(Y_{1}, Y_{2}\right)$.

Proof: Note that when $\vartheta_{12}{ }^{X}=0, \mathrm{X}_{1}$ is a scalar multiple of $\mathrm{X}_{2}$. In that case we have $\mathrm{E} \operatorname{Max}\left(\mathrm{X}_{1}, \mathrm{X}_{2}\right)$ $=\mathrm{E} \operatorname{Max}\left(\mathrm{X}_{1}, \mathrm{kX}_{1}\right)$ which is equal to $\mathrm{EXX}_{1}=0$ (if $\mathrm{k}>1$ ) or $\mathrm{EX}_{1}=0$ (if $\mathrm{k} \leq 1$ ). On the other hand, $\mathrm{E} \operatorname{Max}\left(\mathrm{Y}_{1}, \mathrm{Y}_{2}\right)$ is strictly positive provided $\vartheta_{12}{ }^{Y} \neq 0$. Now since $\operatorname{Cos}\left(\vartheta_{12}{ }^{X}\right)$ is a continuous function of $\vartheta_{12}{ }^{X}$, it follows that for any fixed non-zero value of $\vartheta_{12}{ }^{Y}, \operatorname{E~} \operatorname{Max}\left(\mathrm{X}_{1}, \mathrm{X}_{2}\right)<\mathrm{E}$ $\operatorname{Max}\left(\mathrm{Y}_{1}, \mathrm{Y}_{2}\right)$ for all values of $\vartheta_{12}{ }^{X}$ sufficiently close to zero. This proves our claim for the case when the projects have two paths. The result can easily be extended to projects with an arbitrary number of paths by letting the angles between every pair of rows of the matrix B be sufficiently small. Q.E.D.

The foregoing counterexample was one in which the marginal distributions induced by the joint were different, since the matrices A and B were not identical. For the record, we give a counterexample with discrete distributions in which the marginal distributions are identical. 
$x 2$

\begin{tabular}{|c|c|c|c|}
\hline $\mathbf{X} 1$ & 6 & 7 & 9 \\
\hline 6 & 0.1 & 0.2 & 0.1 \\
\hline 8 & 0.4 & & \\
\hline 9 & & & 0.2 \\
\hline
\end{tabular}

$\begin{array}{lll}0.5 & 0.2 & 0.3\end{array}$

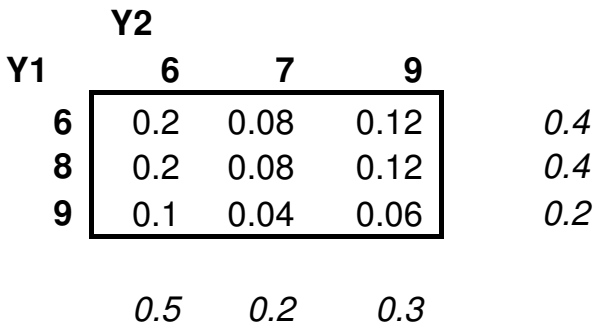

The correlation coefficient of $\mathrm{X}_{1}$ and $\mathrm{X}_{2}$ is 0.167 while $\mathrm{Y}_{1}$ and $\mathrm{Y}_{2}$ are independent.

But $\operatorname{EMax}\left(\mathrm{X}_{1}, \mathrm{X}_{2}\right)=7.9>\mathrm{E} \operatorname{Max}\left(\mathrm{Y}_{1}, \mathrm{Y}_{2}\right)=7.8$

\subsection{The effect of activity correlation on the distribution of path length}

A crucial ingredient of the PERT method is the assumption that the Central Limit Theorem applies to each path of a large project network. However, if the activities on a path are correlated, the path length may not be normally distributed. We report the results of a simulation experiment to demonstrate the non-normality of path length distributions when activity durations are correlated.

Dependent and independent random vectors were created from independent and identical random variables $\mathrm{X}_{1}$ through $\mathrm{X}_{50}$, each distributed uniformly on $[0,1]$. Denote the vector $\left[\mathrm{X}_{1} \ldots \mathrm{X}_{50}\right]$ by $\mathbf{X}$. We generated the vectors $\mathbf{Y}=\mathrm{AX}$ and $\mathbf{Z}=\mathrm{BX}$ via 50 by 50 matrices $\mathrm{A}$ and $\mathrm{B}$. Letting $\mathrm{A}$ be the identity matrix, we obtained a vector $\mathbf{Y}$ with independent components. The sum of the components of $\mathbf{Y}$ is shown in Figure 3; it is approximately bell shaped, as expected. 


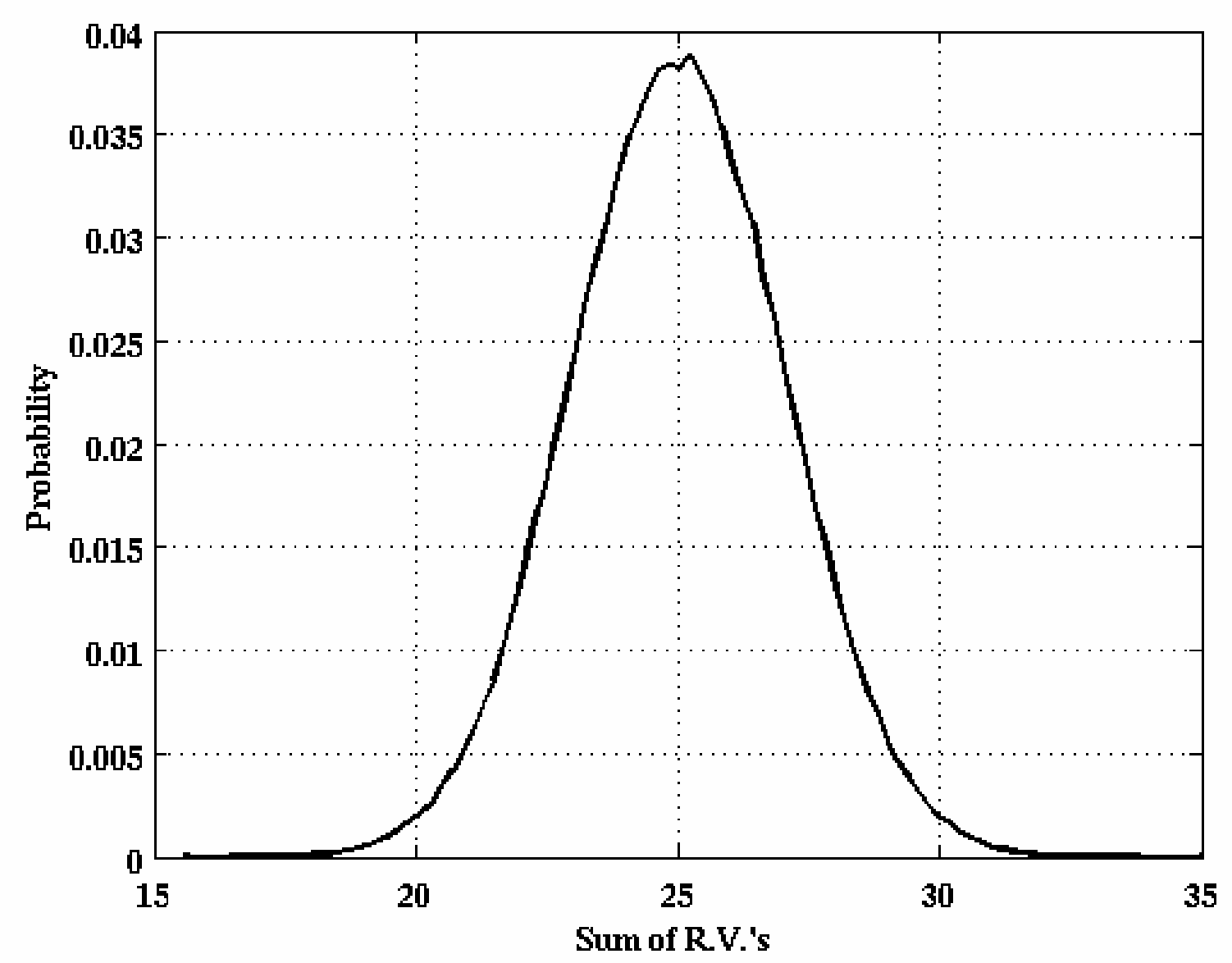

\section{Figure 3}

The elements $b_{\mathrm{ij}}$ of $\mathrm{B}$ were generated as follows. For each row $\mathrm{i}$, the following was done: for $\mathrm{j}=1, \mathrm{~b}_{\mathrm{ij}}$ was sampled uniformly from [0,2]; for all other values of $\mathrm{j}$, $\mathrm{b}_{\mathrm{ij}}$ was sampled uniformly from $[-.5, .5]$. The matrix B was then fixed for the rest of the experiment. Note that the vector $\mathbf{Z}$ generated by B consists of correlated components. Each component represents the completion time of an individual activity and the sum represents the path length. The sum of the 50 component random variables is pictured in Figure 4. Half a million data points were used to generate figures 3 and 4 . The plateau like nature of Figure 4 is clear evidence of the non-normality of the underlying distribution. 


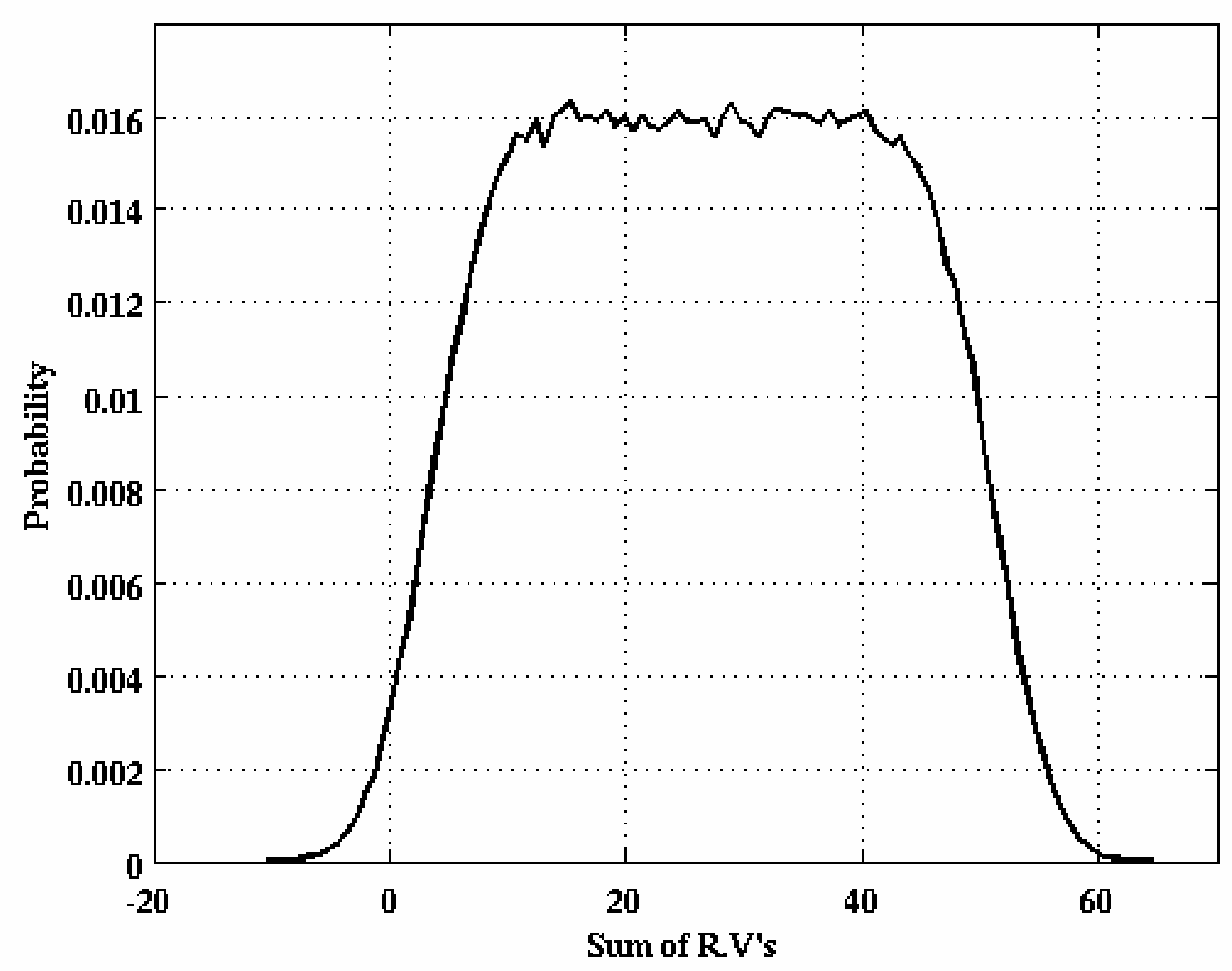

\section{Figure 4}

It is worth noting that some of the elements of the matrix B are guaranteed to be negative, by construction, implying negative correlation between some pairs of activities. An interesting observation from our simulation was the following: the path length showed the most severe departures from the bell shape when the path comprised a mixture of pair-wise positively correlated as well as negatively correlated activities. Recall from the discussion on page 16 that there are practical justifications for modeling correlations of either sign.

We remark in passing that it is a hard theoretical problem to obtain precise results that capture the extent to which the Central Limit Theorem breaks down when the summands are dependent 
random variables. The existing results in this direction do not seem to be relevant to PERT networks (see [1] and [8]).

\subsection{Correlated activity durations and correlated activity completion times}

Ringer [10] developed formulae for the distribution functions of serial and parallel projects with correlated activity completion times, where the completion time of an activity is the point in time at which it finishes as opposed to its duration. (Recall that a serial project is one in which the activities are required to be completed one after the other because of precedence constraints while a parallel project is one in which all the activities start simultaneously because there are no precedence constraints between them.) We ask: are pair-wise positively correlated activity completion times equivalent to pair-wise positively correlated activity durations? Theorem 5 partially answers the question.

\section{Theorem 5}

If activity durations are pairwise positively correlated, then so are activity completion times provided the activity durations form a multivariate normal vector.

Proof: Let $\mathbf{A}=\left(\mathrm{A}_{1}, \mathrm{~A}_{2}, \ldots, \mathrm{A}_{\mathrm{N}}\right)$ and $\mathbf{C}=\left(\mathrm{C}_{1}, \mathrm{C}_{2}, \ldots, \mathrm{C}_{\mathrm{K}}\right)$ denote vectors of activity durations times and activity completion times, respectively. Each $C_{i}$ is a coordinatewise increasing function of $\mathbf{A}$. Hence it follows from a standard result on association that if $\mathbf{A}$ is associated, so is $\mathbf{C}$. Now a theorem of Pitt [6] states that a normal random vector is associated if and only if it is pairwise positively correlated. The theorem follows. Q.E.D 


\section{Concluding Remarks}

The rule of thumb 'greater correlation implies smaller PERT bias' holds true for projects with joint distributions that are spatially symmetric is a strong sense; these joint distributions induce marginal distributions (that may be viewed as the distributions of path lengths) that are symmetric. The rule breaks down when path lengths have asymmetric distributions. An exception is when paths are very highly correlated; in that case, the rule of thumb does hold irrespective of the shapes of the distributions of path durations.

Theorem 2 of this paper may be viewed as a correlated-activity counterpart of an independentactivity result on the impact of increasing activity variance on PERT bias. It has been shown, using stochastic ordering tools, that increasing activity variance holding mean durations constant inflates PERT bias in a network with independent activities for several commonly encountered classes of probability distributions (but not all; see Gutierrez and Paul [5] for counterexamples). Note that Theorem 2 is a result on PERT bias in which the mean and covariance vectors are fixed, and the variance vector is perturbed. In the present paper, we examine the impact on PERT bias when the mean and the variance vectors are fixed and the covariance matrix is perturbed. It is interesting to note that neither result is distribution-free.

\section{Acknowledgements}

We would like to thank Haldun Aytug for his constructive comments during our work on this paper. 


\section{References}

[1] J.Dedecker, F. Merlevède. "Necessary and sufficient conditions for the conditional central limit theorem", Annals of Probability, 30, no. 3 (2002), 1044-1081.

[2] B.M.Dodin. "Bounding the Project Completion Time Distribution in PERT Networks," Operations Research, 33 (1985), 862-881.

[3] S.E.Elmaghraby. Activity Networks: Project Planning and Control by Network Models. John Wiley and Sons, New York, 1977.

[4] J.D.Esary, F.Proschan, D.Walkup. "Association of random variables, with applications", Annals of Mathematical Statistics, 38 (1967), 1466-1474.

[5] G.Gutierrez, A.Paul. "Analysis of the Effects of Uncertainty, Risk-Pooling, and Subcontracting Mechanisms on Project Performance”, Operations Research, 48 (2000), no. 6, 927-938.

[6] K.Joag-Dev, M.Perlman, L.Pitt. “Association of Normal random variables and Slepian's inequality", Annals of Probability, 11 (1983), no. 2, 451--455.

[7] K.R.MacCrimmon, C.A.Ryavec. "An Analytical Study of the PERT Assumptions," Operations Research, 1964, vol 12 issue 1, 16-37.

[8] D. L. McLeish. "Dependent Central Limit Theorems and Invariance Principles", Annals of Probability, 1974, 2, 620-628.

[9] L.D.Pitt, "Positively Correlated Normal Variables are Associated," Annals of Probability, 1982, 10 (2), 496-499.

[10] L.J.Ringer. "A Statistical Theory for PERT in which Completion Times of Activities are Dependent," Management Science, Vol.17, No.11, 1971.

[11] R.Slowinski and J.Weglarz (editors). Advances in Project Scheduling. Elsevier, 1989. 